\title{
Rice Starch can be Used as a Drug During Treatment the Colon and Rectum
}

\author{
Hassan Ababneh* \\ $R \& D$ Department, Jordan
}

*Corresponding Author: Hassan Ababneh, R\&D Department, Jordan.

Received: July 17, 2019; Published: July 24, 2019

DOI: 10.31080/ASPS.2019.03.0353

Anxiety and depression are highly comorbid disorders possibly sharing a common neurobiological mechanism. The dysfunction of serotoninergic, noradrenergic and dopaminergic neurotransmission, abnormal regulation in the hypothalamic-pituitary-adrenal axis (HPA), disturbance of cellular plasticity including reduced neurogenesis, or chronic inflammation connected with high oxidative damage play a crucial role in the development of anxiety and depression. The present study was aimed to investigate the effects of atenolol alone and in combination with alprazolam/escitalopram on anxiety, depression and oxidative stress. Wistar albino rats were subjected to $21^{\text {st }}$ day treatment of drugs then exposed to elevatedplus maze (EPM) and modified forced swim test (MFST), and oxidative stress markers were estimated in isolated brain tissue of all groups. The results indicated that atenolol in combination with alprazolam/escitalopram exhibited antidepressant effects by significantly decreasing the immobility and increasing the swimming behavior in the MFST and anti-anxiety effects by increasing the percentage preference and number of open arm entries as well as time spent in open arm in EPM. Pre-treatment with atenolol alone and combination with alprazolam/escitalopram also ameliorated tissue glutathione (GSH) and decreased malondialdehyde (MDA) level significantly which explore antioxidant properties of drugs, and combination augments the therapeutic response of monotherapy in depression. In conclusion behavioral and biological findings indicate that the combination of atenolol with alprazolam/escitalopram has the potential of being highly efficacious in treating anxiety and depressive disorders as well as oxidative stress.
Anxiolytic dose of atenolol exhibits antioxidant and antidepressant effect.

Alprazolam/escitalopram with atenolol protects brain tissue from oxidative damage.

Atenolol with alprazolam/escitalopram offers advantage over individual treatment.

\section{Volume 3 Issue 8 August 2019 \\ (C) All rights are reserved by Hassan Ababneh.}

